\title{
Robust and Compositional Verification of Object Capability Patterns
}

\author{
DAVID SWASEY, MPI-SWS, Germany \\ DEEPAK GARG, MPI-SWS, Germany \\ DEREK DREYER, MPI-SWS, Germany
}

In scenarios such as web programming, where code is linked together from multiple sources, object capability patterns (OCPs) provide an essential safeguard, enabling programmers to protect the private state of their objects from corruption by unknown and untrusted code. However, the benefits of OCPs in terms of program verification have never been properly formalized. In this paper, building on the recently developed Iris framework for concurrent separation logic, we develop OCPL, the first program logic for compositionally specifying and verifying OCPs in a language with closures, mutable state, and concurrency. The key idea of OCPL is to account for the interface between verified and untrusted code by adopting a well-known idea from the literature on security protocol verification, namely robust safety. Programs that export only properly wrapped values to their environment can be proven robustly safe, meaning that their untrusted environment cannot violate their internal invariants. We use OCPL to give the first general, compositional, and machine-checked specs for several commonly-used OCPs-including the dynamic sealing, membrane, and caretaker patterns-which we then use to verify robust safety for representative client code. All our results are fully mechanized in the Coq proof assistant.

CCS Concepts: • Security and privacy $\rightarrow$ Security requirements; $\bullet$ Theory of computation $\rightarrow$ Program specifications; Separation logic;

Additional Key Words and Phrases: object capabilities, robust safety, separation logic, logical relations, compositional verification

\section{ACM Reference Format:}

David Swasey, Deepak Garg, and Derek Dreyer. 2017. Robust and Compositional Verification of Object Capability Patterns. Proc. ACM Program. Lang. 1, OOPSLA, Article 89 (October 2017), 26 pages. https://doi.org/ $10.1145 / 3133913$

\section{INTRODUCTION}

Suppose you have a mutable reference $\ell$ whose contents you care about, meaning that you want to impose some invariant on it (e.g., $\ell$ always points to an even number). Suppose further that you want to share access to $\ell$ with code you did not write and that you do not trust to preserve the invariant on $\ell$. To ensure the invariant on $\ell$ is maintained, you therefore do not want to pass the untrusted code the reference $\ell$ directly. Instead, you might construct a read-only wrapper $w$ as follows:

$$
\text { readonly } \triangleq \lambda r . \lambda_{-} . ! r \quad w \triangleq \text { readonly } \ell
$$

Authors' addresses: MPI-SWS, Saarland Informatics Campus (SIC), Campus E1.5, 66123 Saarbrücken, Germany, \{swasey, dg, dreyer\}@mpi-sws.org.

This work is licensed under a Creative Commons Attribution 4.0 International License.

(C) 2017 Copyright held by the owner/author(s).

2475-1421/2017/10-ART89

https://doi.org/10.1145/3133913

Proceedings of the ACM on Programming Languages, Vol. 1, No. OOPSLA, Article 89. Publication date: October 2017. 
Here, readonly transforms a reference $r$ into a thunk that, when applied, returns the current contents of $r$. The expression $w$ applies readonly to our reference of interest $\ell$, constructing a function for reading $\ell$ 's contents. You can now pass $w$ to untrusted code without worrying about it corrupting your invariant on $\ell$.

Wrappers like $w$ are often called object capabilities, and the wrapper construction function readonly is a very simple example of an object capability pattern (OCP) [Miller et al. 2000]. Although OCPs date back at least to the 1970s [Morris 1973], they have gained increased currency in recent years, both in new languages centered around object capabilities [Miller et al. 2000; Mettler et al. 2010; Spiessens and Roy 2004; Stiegler and Miller 2006] and in the context of web programming, where interfacing with untrusted code is commonplace. Web sandboxing systems like Yahoo!'s ADsafe [Crockford 2008; Politz et al. 2014] and Google's Caja [Miller et al. 2008], for example, elaborate untrusted JavaScript source code into an ostensibly safe subset of JavaScript while introducing wrappers that attenuate access to JavaScript libraries. Caja relies on the so-called membrane pattern, which automatically wraps all objects crossing to untrusted, sandboxed code so that access to the underlying objects is appropriately restricted.

OCPs are believed to provide crucial security guarantees because, in a language like JavaScript, objects provide essentially no data abstraction on their own-the only way to enforce data abstraction is to hide private state in the environment of a closure (e.g., as $\ell$ was hidden in the environment of readonly $\ell$ in the example above). So OCPs, which use closures to mediate access to private state in a systematic way, are one of the few effective mechanisms available in a language like JavaScript for enforcing data abstraction in the presence of possibly malicious code.

Unfortunately, despite the ubiquity of OCPs in modern web programming, remarkably little attention has been paid to the question of what exactly are the security guarantees that such OCPs are supposed to provide, and how might we prove that they actually provide them. Even in the case of the extremely basic readonly pattern shown above, it is not at all obvious what is the "right" formal specification for readonly. What, in particular, are the formal conditions on $\ell$ that are needed to guarantee that readonly $\ell$ can be "safely" shared with untrusted code? If $\ell$ merely points to an integer, no conditions may be necessary, but what if $\ell$ points to a closure or some other higher-order object? How do we know that giving readonly access to $\ell$ will not indirectly give untrusted code a way of gaining full access and violating important invariants maintained by the user of this OCP?

The most recent, state-of-the-art attempt to grapple with these types of questions is due to Devriese et al. [2016]. They build a Kripke logical relations model for reasoning about object capabilities in a language with higher-order state, and they use their model to verify several concrete examples of capability-wrapped user code. In each case, they demonstrate that invariants on the private state of the user code are preserved even when the user code is shared with unknown attacker code. However, their model is limited in that it provides no way to compositionally specify what an OCP does, and it provides no clear specification of the general property that a piece of user code must satisfy in order to be safely shareable with untrusted code. Furthermore, they only consider very simple capability patterns, none as complex as, say, the aforementioned membrane pattern.

In this paper, we present OCPL (a Logic for $\boldsymbol{O C P}$ ), the first formal system for compositionally specifying and verifying the security guarantees provided by OCPs, in the context of a simple but representative programming language with higher-order functions, state, and concurrency. ${ }^{1}$ In contrast to prior work, OCPL enables one to reason modularly about both OCP implementations and user code that depends on them, and to specify a general property on user code that ensures such code can be safely shared with untrusted code without having its internal invariants violated.

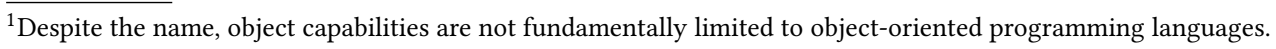


We use OCPL to reason about several commonly-used OCPs, including the dynamic sealing (sealerunsealer), membrane, and caretaker patterns, and in so doing, provide the first formal explanation of what these OCPs achieve.

OCPL is a program logic derived from Iris, a recently developed framework for higher-order concurrent separation logic [Jung et al. 2015, 2016; Krebbers et al. 2017a]. Iris was originally proposed as a very general logic with a few simple primitives, using which one can derive advanced proof principles from a variety of modern separation logics as needed. Moreover, Krebbers et al. [2017b] have recently developed a powerful new proof mode for Iris in the Coq proof assistant. The Iris proof mode enables one to carry out interactive, tactical, machine-checked proofs about programs in the Iris logic (embedded in Coq) in much the same way as one normally carries out interactive tactical proofs when working in the meta-logic of Coq itself. By virtue of building OCPL on top of Iris, we inherit the flexibility of the existing Iris framework, as well as its support for mechanizing proofs about programs in Coq. All our results and examples are, in fact, fully verified in Coq. ${ }^{2}$

The key idea in OCPL is how it characterizes the interface between verified user code and untrusted code, via the concept of a "low-integrity value" (or low value for short) adapted from the literature on verification of security protocols [Abadi 1999]. Roughly speaking, a low value is a value that can be safely shared with untrusted code, such as the closure wrappers returned by OCPs. More precisely, a low value is a value from which no code can possibly extract a direct reference to private state. To formalize this notion, we employ a logical relation, which is easy to define using Iris's built-in support for guarded recursive predicates. Then, with the idea of low values in hand, it becomes clear how to specify when a piece of user code can be safely linked with untrusted code-namely, when the only values passed back and forth between them are low values.

To make things concrete, let us return to our motivating example. Using the notion of low values, we can prove the following specification for the readonly pattern:

$$
\forall \ell .\{T\} ! \ell\{x \text {. lowval } x\} \Rightarrow\{T\} \text { readonly } \ell\{f \text {. lowval } f\}
$$

The premise of this spec says that, in order to apply readonly to a location $\ell$, we must first prove that dereferencing $\ell$ always produces a low value. (This makes sense because once we pass the read-only wrapper to untrusted code, it may invoke the wrapper and obtain the contents of $\ell$ at any time.) The payoff is in the conclusion: the closure returned by readonly $\ell$ is itself a low value and may therefore be safely shared with untrusted code.

Given this specification for readonly, let us now consider a client of readonly that wishes to maintain an invariant on its private (i.e., high-integrity) state:

$$
\begin{aligned}
\text { usetwo } \triangleq & \text { let } r=\text { ref } 2 \text { in } \\
& \text { let } w=\text { readonly } r \text { in } \\
& \text { let } u s e=\lambda_{-} \text {. assert }((! r)=2) \text { in } \\
& (\text { use, } w)
\end{aligned}
$$

Here, the expression usetwo allocates a reference cell $r$ containing the value 2, and returns a pair of functions, one of which simply asserts that $r$ continues to contain 2, and the other of which is the result of readonly $r$. The reference cell $r$ is, however, kept private (i.e., hidden in the environments of the closures use and $w$ ).

Our aim is to verify that usetwo can be safely linked with arbitrary untrusted code. To do this, we first prove that it satisfies the spec $\{T\}$ usetwo $\{x$. lowval $x\}$ which means that we can always run usetwo and, if we do, it returns a low value. It is easy to see intuitively why this spec holds:

\footnotetext{
${ }^{2}$ The Coq formalization and a version of this paper with appendices are available online [OCPL 2017].
} 
reference cell $r$ always contains 2, which is trivially low, so together with the spec for readonly, this is sufficient to ensure that the closures returned by usetwo are low as well.

Next, we appeal to a general meta-theorem-and one of the main technical results of this paper-called robust safety. Robust safety states that, if some user code satisfies a spec like the one given above for usetwo-i.e., a spec whose postcondition stipulates that the resulting value is low-integrity-then we can run that verified user code under an arbitrary adversarial context $C$ (i.e., any context $C$ that does not itself contain any assert statements), and we will be assured that the execution of the resulting program will never result in a violation of any of the user code's internal assertions. Thus, in the particular case of usetwo, we know that the assertion that $r$ equals 2 will never fail.

Robust safety is a well-known meta-theorem in the security literature [Bengtson et al. 2011; Gordon and Jeffrey 2001], but it has not heretofore been employed in the context of object capability programming. One of the central contributions of this paper is the observation that robust safety is exactly the property a language must satisfy in order to support OCPs. Moreover, as we will demonstrate via a range of interesting examples, the notion of low-integrity values is essential to compositionally specifying an OCP's contribution toward the robust safety of programs that use it.

The remainder of the paper is structured as follows. First, in §2, we introduce some basics of OCPL, along with our formalization of low values, explain how we verify our motivating readonly example, and state the key metatheorems of adequacy and robust safety. In $\S \S 3-5$, we present specifications for several more complex and realistic OCPs, along with examples of how to use those specifications to verify representative clients. In $\S 6$, we discuss related work. Finally, in $\S 7$, we conclude with a discussion of future work.

\section{ROBUST SAFETY AND OCPL}

In this section, we define the semantics of the programming language we will be reasoning about (\$2.1), present the main ideas of OCPL and show how to use it to reason formally about our motivating example (§2.2), and state key metatheorems for OCPL (§2.3).

\subsection{A Higher-Order Concurrent Heap Language with Assertions}

The programming language we consider in this paper, which we call HLA, is a fairly standard higher-order concurrent imperative language, essentially the same as the one studied by Krebbers et al. [2017a], but with one important extension. We define the language in Fig. 1. The core of the language is a call-by-value $\lambda$-calculus with recursive functions, products, sums, references, and fork-based concurrency. ${ }^{3}$ The definitions of free and bound variables and capture-avoiding substitution are standard and omitted. We endow the language with an operational semantics in the style of Felleisen and Hieb [1992]. The head reduction judgment $e ; \sigma \rightarrow_{\mathrm{r}} e^{\prime} ; \sigma^{\prime} ; \overrightarrow{e_{f}}$ describes the single steps of computation that may be taken by individual threads. It means that, starting in state $\sigma$, a thread running expression $e$ may step to expression $e^{\prime}$ while changing the state to $\sigma^{\prime}$ and forking off a list of threads running expressions $\overrightarrow{e_{f}}$. Threadpool reduction $T ; \sigma \longrightarrow T^{\prime} ; \sigma^{\prime}$ lifts head reduction to lists of concurrently running threads. We leave implicit the choice of operators unop and binop, but note that they reduce according to (partial) evaluation functions and are subject to a semantic constraint; for example, the reduction rule for unary operators

$$
\text { unopv; } \sigma \rightarrow_{\mathrm{r}} v^{\prime} ; \sigma ; \varepsilon \quad \text { if eval unop } v=v^{\prime}
$$

employs a partial function eval $: U n o p \rightarrow$ Val $\rightarrow$ Val (which, for the robust safety theorem presented below, is assumed to send low-integrity values to low-integrity values). As is standard in

\footnotetext{
${ }^{3}$ OCPs are typically studied in the context of an event-driven programming model. We work in the setting of fork-based concurrency because Iris supports it natively and it does not seem to significantly affect reasoning about OCPs.
} 
Lit $\ni$ lit $::=n \mid b$

$\operatorname{Exp} \ni e::=x|\operatorname{rec} f x . e| e e \mid$ lit $\mid$ unope $\mid$ binope $e \mid$ if $e$ then $e$ else $e \mid$

()$|(e, e)|$ fst $e \mid$ snd $e \mid$ inl $e \mid$ inr $e \mid$ case $e$ of inl $x \Rightarrow e \mid$ inr $x \Rightarrow e \mid$

fork $e|\ell|$ ref $e|! e| e \leftarrow e \mid$ CAS e e e $\mid$ assert $e$

Val $\ni v::=\operatorname{rec} f x . e \mid$ lit $|()|(v, v) \mid$ inl $v \mid$ inr $v \mid \ell$

Ectx $\ni K::=$ (standard left-to-right evaluation contexts)

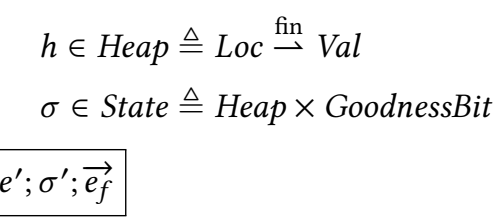

$$
\begin{gathered}
g \in \text { GoodnessBit } \triangleq\{\text { OK, Fail }\} \\
T \in T \text { Tpool } \triangleq \text { Exp }^{*}
\end{gathered}
$$

$e ; \sigma \rightarrow_{\mathrm{r}} e^{\prime} ; \sigma^{\prime} ; \overrightarrow{e_{f}}$

Head reduction (selected rules)

$$
\begin{aligned}
& (\operatorname{rec} f x . e) \text { closed } \\
& \overline{(\operatorname{rec} f x . e) v ; \sigma \rightarrow_{\mathrm{r}} e[v / x, \operatorname{rec} f x . e / f] ; \sigma ; \varepsilon} \\
& \text { assert false; }(h, g) \rightarrow_{\mathrm{r}}() ;(h, \text { Fail }) ; \varepsilon \quad \text { fork } e ; \sigma \rightarrow_{\mathrm{r}}() ; \sigma ; e \\
& \operatorname{ref} v ;(h, g) \rightarrow_{\mathrm{r}} \ell ;(h \uplus[\ell \mapsto v], g) ; \varepsilon \quad \frac{\sigma \cdot h(\ell)=v}{! \ell ; \sigma \rightarrow_{\mathrm{r}} v ; \sigma ; \varepsilon} \quad \frac{h(\ell)=v}{\ell \leftarrow w ;(h, g) \rightarrow_{\mathrm{r}}() ;(h[\ell \mapsto w], g) ; \varepsilon} \\
& \frac{\sigma . h(\ell) \neq v_{1}}{\mathrm{CAS} \ell v_{1} v_{2} ; \sigma \rightarrow_{\mathrm{r}} \text { false } \sigma ; \varepsilon} \quad \text { CAS } \ell v_{1} v_{2} ;\left(h \uplus\left[\ell \mapsto v_{1}\right], g\right) \rightarrow_{\mathrm{r}} \text { true; }\left(h \uplus\left[\ell \mapsto v_{2}\right], g\right) ; \varepsilon \\
& T ; \sigma \longrightarrow T^{\prime} ; \sigma^{\prime} \\
& \text { Threadpool reduction } \\
& \frac{e ; \sigma \rightarrow_{\mathrm{r}} e^{\prime} ; \sigma^{\prime} ; \overrightarrow{e_{f}}}{\left(T_{1}, K[e], T_{2}\right) ; \sigma \longrightarrow\left(T_{1}, K\left[e^{\prime}\right], T_{2}, \overrightarrow{e_{f}}\right) ; \sigma^{\prime}}
\end{aligned}
$$

Fig. 1. Heap language with assertions

semantics for languages with mutable references, locations $\ell$ are not part of the "surface" syntax of the language; they may only arise dynamically during execution. Using compare-and-swap (CAS), one can implement locks (and other concurrency primitives). ${ }^{4}$ We use the symbol "' ' in place of a bound variable to indicate any variable not occurring free in the scope of the binder. In addition to standard derived forms (e.g., $n$-ary products and let expressions), we write

$$
\begin{aligned}
\lambda x . e & \triangleq \operatorname{rec}_{-} x . e \\
\lambda x_{1} \cdots x_{n} . e & \triangleq \lambda x_{1} \cdot \cdots \cdot \lambda x_{n} \cdot e \\
\operatorname{rec} f x_{1} \cdots x_{n} . e & \triangleq \operatorname{rec} f x_{1} \cdot \lambda x_{2} \cdots x_{n} . e
\end{aligned}
$$

\footnotetext{
${ }^{4}$ The expression CAS $\ell v_{1} v_{2}$ atomically compares the contents of location $\ell$ to value $v_{1}$ and, if equal, writes $v_{2}$ to $\ell$ and returns true; otherwise, it returns false. For simplicity, we do not restrict CAS to comparing first-order, "word-sized" values. This is unrealistic, but we use CAS in only realistic ways. (Spinlocks, in particular, are compatible with such a restriction and suffice for our purposes.)
} 
and we use pattern-matching notation to deconstruct products, rather than a series of fst and snd projections.

The one new feature in HLA, not present in Krebbers et al.'s calculus, is assertion expressions, assert $e$, which serve to specify safety properties that should always hold dynamically. Assertions in HLA operate a little differently from assertions in languages like $\mathrm{C}$ or Java, where failed assertions abort execution or raise an exception. ${ }^{5}$ Here, $e$ is a boolean expression. If $e$ evaluates to true, then assert $e$ has no effect. But if $e$ evaluates to false, then assert $e$ has the effect of irreversibly setting a "goodness bit" $g$, which is maintained as part of the machine state, to Fail. (The bit is initially set to OK.) This goodness bit simply checks whether any assertion expression has dynamically failed during execution. Ultimately, our robust safety theorem will use this goodness bit to ensure that, for properly verified code, the goodness bit will remain OK throughout execution (and hence all dynamic assert checks must succeed).

The reader may wonder why we employ a goodness bit instead of just saying that assert false gets stuck or aborts the program. The reason is simple: we wish to use OCPL to reason not only about fully verified code but also about the behavior of verified code when linked with untrusted code (that does not itself contain assertions). So it is important that we have a way of verifying Hoare triples even for code that may very well get stuck (i.e., fail to make progress) thanks to dynamic type errors introduced by the untrusted code. We nevertheless want to say that assert expressions in such code always succeed, so we must differentiate assert false from other stuck states. ${ }^{6}$

\subsection{A Program Logic for Reasoning about OCPs}

We now present some of the essential features of OCPL, our logic for reasoning about OCPs.

Progressive vs. non-progressive triples. OCPL is a Hoare-style program logic derived from Iris, a modern separation logic for higher-order concurrent imperative programs. ${ }^{7}$ As such, one of the main assertions in OCPL is the Hoare triple, which is used to specify the behavior of expressions in terms of preconditions and postconditions. The Hoare triple $\{P\} e\{x . Q\}_{p}$ asserts that, assuming $e$ is executed starting in a state satisfying the precondition $P$, then it will execute without any dynamically failing assert expressions, and if it terminates with value $v$, then the final state will satisfy the postcondition $Q$ (which may mention the bound variable $x$ ). The progress bit $p \in\{$ progress, noprogress\} indicates whether the triple is progressive (i.e., ensures that $e$ does not get stuck) or non-progressive. ${ }^{8}$ As shorthand, we will write $\{P\} e\{x . Q\}$ and $\{P\} e\{x . Q\}_{\text {? }}$ for progressive and non-progressive triples, respectively; the former assertion implies the latter. We often write the postcondition of a Hoare triple as $\left\{x_{1} \cdots x_{n}\right.$, ret pat. $\left.Q\right\}$, which binds the $x_{i}$ and specifies that any returned value will be precisely pat. This notation is (roughly) syntactic sugar for the post-condition $\left\{x . \exists x_{1}, \ldots, x_{n} . x=p a t * Q\right\}$.

Hoare triples in Iris (and thus OCPL) enjoy the standard structural rules for higher-order concurrent separation logic (e.g., the rule of consequence, the frame rule, and monadic bind and return) as well as rules for defining and enforcing logical protocols on physical and ghost state. Readers interested in these aspects of OCPL should consult Jung et al. [2015] for a high-level overview and Jung et al. [2017] for a recent account of Iris's foundations.

\footnotetext{
${ }^{5}$ We define C-style assertions-which we call assumption expressions-in $\S 3$.

${ }^{6}$ Instrumenting the state with a goodness bit is not strictly necessary; for example, one could alternatively use a special machine configuration to indicate failed assertions.

${ }^{7}$ Readers unfamiliar with separation logic can, to a first approximation, read separating conjunction $P * Q$ as conjunction $P \wedge Q$ and magic wand $P * Q$ as implication $P \Rightarrow Q$

${ }^{8}$ Note: this is a point of difference from the original Iris, which only supported progressive triples.
} 


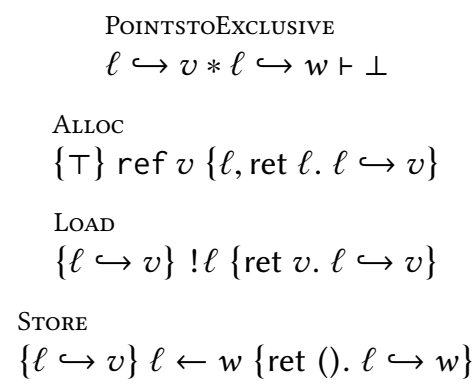

(a) High locations

\author{
HighNotLow \\ $\ell \hookrightarrow v *$ lowloc $\ell \vdash \perp$ \\ AllocLow \\ $\{$ lowval $v\}$ ref $v\{\ell$, ret $\ell$. lowloc $\ell\}$ \\ LOADLOW \\ $\{$ lowloc $\ell\} ! \ell\{x$. lowval $x\}$ \\ STORELOW \\ $\{$ lowloc $\ell *$ lowval $v\} \ell \leftarrow v\{\operatorname{ret}() . \top\}$
}

(b) Low locations

Fig. 2. Selected proof rules for locations

In addition, OCPL enjoys a full set of basic rules for HLA, comprising progressive triples for reasoning about head reductions and non-progressive triples for reasoning about stuck expressions. The following three rules, for example, concern application.

$$
\begin{aligned}
& \text { ApP }
\end{aligned}
$$

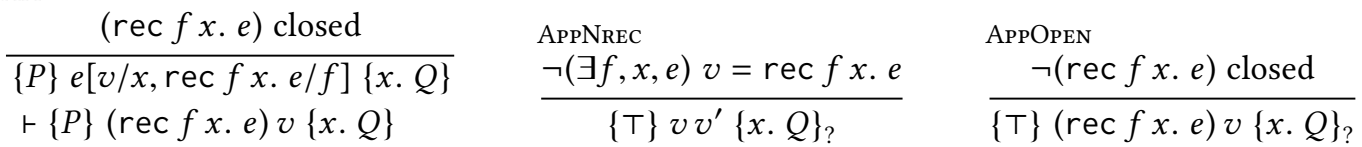

Rule Apr says that, to prove a triple for a closed function application, it suffices to show an analogous triple after $\beta$-reduction. Rules AppNrec and AppOpen give non-progressive triples to applications that, according to the operational semantics of HLA, always get stuck: as such applications never produce a value, they can be given an arbitrary postcondition $Q$.

We omit analogous rules for other expressions that ignore the ambient machine state, turning now to the more interesting rules for assertions and locations.

Assertion expressions. OCPL supports the following rule for assertion expressions.

$$
\begin{aligned}
& \text { Assert } \\
& \{P\} \text { e }\{x . x=\text { true } * Q\}_{p} \vdash\{P\} \text { assert } e\{\text { ret }() . Q\}_{p}
\end{aligned}
$$

AsSERT enables reasoning about successful assertions. It says that, to prove a Hoare triple for assert $e$ with postcondition $Q$ and return value (), it suffices to prove that $e$ evaluates to true with postcondition $Q$.

High vs. low locations. OCPL divides memory locations (i.e., mutable references) into two types: high-integrity and low-integrity (or just high and low for short). High locations are locations that are private to user code, on which it may place invariants of its choosing, and to which untrusted code should not be given direct, unfettered access. Low locations are locations that may be freely shared with-and may in fact have been allocated by-untrusted code. Note that there is no distinction between high and low locations in the operational semantics of HLA; rather, this distinction is merely something we track in OCPL in order to formally specify the interface between user code and untrusted code.

Reasoning about high locations. Since high locations are locations controlled privately by verified user code, OCPL supports reasoning about them in essentially the same way as one reasons about pointers in traditional separation logic-via the classic "points-to" assertion $\ell \hookrightarrow v$. This points-to assertion denotes the knowledge that location $\ell$ currently points to value $v$ as well as ownership 


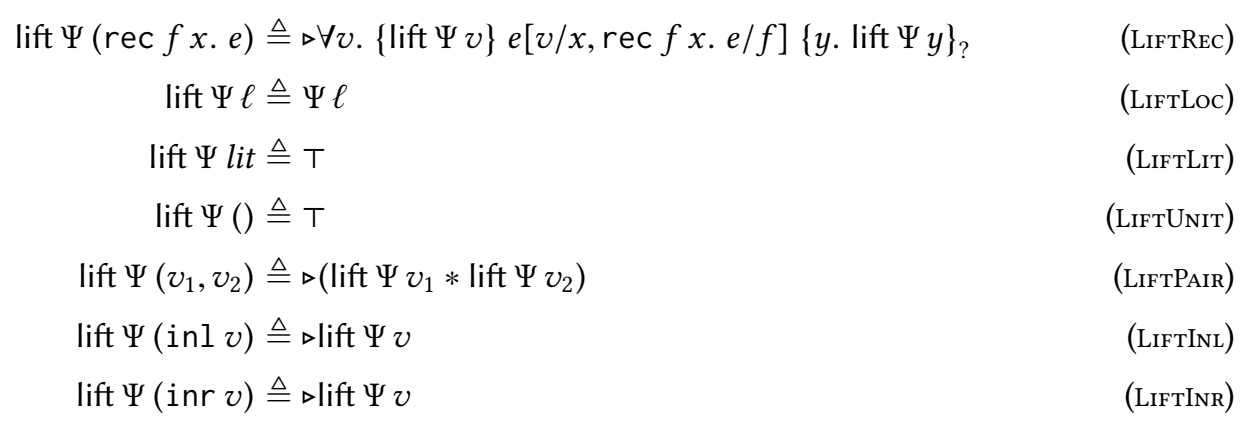

Fig. 3. Lifting location predicates to value predicates

of $\ell$. What this means is that if we own $\ell$, then no other party (e.g., neither another thread nor the current thread's context) can be simultaneously making any assertion about $\ell$, so we should have permission to freely read from and write to $\ell$ without worrying about violating any other party's reasoning. This reasoning is formalized with standard rules about high locations displayed in Fig. 2a.

Reasoning about low locations. We represent the knowledge that a location $\ell$ is low via the assertion lowloc $\ell$. In contrast to the points-to assertion for high locations, the assertion lowloc $\ell$ does not denote any ownership of $\ell$ because as soon as a location is considered safe to be shared with untrusted code, there is no way of knowing what that code will do with it. Rather, lowloc $\ell$ merely tells us that $\ell$ is low now and will remain low forever. Moreover, if $\ell$ is low-and hence safely shareable with untrusted code-then we know that the value $v$ it points to must also be safely shareable with untrusted code-i.e., $v$ is a low value.

Of course, this begs the question: what is a low value? Intuitively, a low value is a value from which there is no way to extract a high location. We will make this more precise in a moment. For now, assume we have a predicate lowval $v$, which says that $v$ is a low value.

The description of low locations given above is formalized in the proof rules of Fig. $2 \mathrm{~b}$. Rule HighNotLow says that a location cannot be high and low at the same time. Rules AllocLow, LoadLow, and STORELow mimic the corresponding rules for allocation, reading, and writing of high locations, except that for low locations, we do not track the precise contents of the location $\ell$-we merely insist that $\ell$ always points to a low value.

Lifting low locations to low values. We return now to the question of what it means for a value $v$ to be low. Intuitively, a low value is one from which the language constructs of HLA provide no way to get direct access to any high location. This is fundamentally an extensional property, i.e., a property about the observations that a program can make (i.e., the information it can extract) when passed the value $v$. As such, for those readers familiar with classic techniques from program semantics, it will not come as a surprise that a natural way of formally accounting for this property is via a logical relation.

Fig. 3 shows the definition of this logical relation, presented in a somewhat more general form, lift $\Psi v$. Essentially, lift takes as input $\Psi$, a predicate on locations, and lifts it to a predicate on values, with the property that lift $\Psi v$ is true if $\Psi$ holds for any location that can be extracted from $v$. Given this definition, lowval $v$ can be defined simply as lift lowloc $v-i . e$., a value is low if any location that can be extracted from it is low. 
On literals and unit, lift $\Psi$ is trivially true since no location can be extracted from them; on locations, lift $\Psi$ is simply $\Psi$; and on products and sums, lift $\Psi$ is defined in the obvious recursive manner. The only interesting case is the one for functions. For a function value $f$, we want to say that lift $\Psi f$ if, whenever we apply $f$, the resulting term only ever produces values that satisfy lift $\Psi$ (This property on applications of $f$ is expressed via a non-progressive Hoare triple since $f$ may be applied to arguments constructed by untrusted code.) But what arguments should we consider when applying $f$ ? This is where the logical relation comes in: we need only consider argument values that themselves satisfy lift $\Psi$, because ultimately we will make sure that all values passed to untrusted code satisfy lift $\Psi$.

Note that the definition of lift $\Psi$ in the function case is rather circular, since it quantifies over values that satisfy lift $\Psi$. To ensure that this definition is well-founded, we rely on OCPL's support for guarded recursive definitions (inherited from Iris). In OCPL, a definition may be arbitrarily recursive so long as any recursive references are guarded by an occurrence of the $\triangleright$ ("later") modality. Under the hood, all assertions in OCPL are implicitly indexed by steps of computation-i.e., the model of OCPL is a kind of step-indexed model [Birkedal et al. 2011]-and $\triangleright P$ means that $P$ holds, roughly speaking, "after one step of computation". Since the fine points of the "later" modality are not particularly relevant to our main contribution, and our use of "later" is standard, we refer the reader to prior work for a more detailed presentation [Appel et al. 2007].

Using the aforementioned structural and basic rules, we can readily derive rules for working with lifted values. For example, the derived rule

$$
\begin{aligned}
& \text { LiftApp } \\
& \left\{\operatorname{lift} \Psi v * \operatorname{lift} \Psi v^{\prime}\right\} v v^{\prime}\{x . \operatorname{lift} \Psi x\}_{\text {? }}
\end{aligned}
$$

says that one can always apply a lifted value to a lifted argument, and such applications either get stuck (the rule uses a non-progressive triple) or produce a lifted result. These derived rules help, for instance, in verifying clients of the public membrane (§5), which we specify using lift.

To make concrete this rather abstract tour of OCPL, we return to our motivating example. To verify that readonly satisfies it specification, we have to show that the function it returns, $\lambda_{-} . ! \ell$, is a low value given a triple $\{T\} ! \ell\{x$. lowval $x\}$ for dereferencing $\ell$. This follows easily by LiftRec.

To verify that the expression usetwo returns a low value, we proceed as follows. From the allocation ref 2 we obtain $\ell \hookrightarrow 2$ for some location $\ell$ (by Alloc). Since we own $\ell$, we are free to transfer ownership of $\ell$ into a shared invariant, which in Iris (and OCPL) is written $\ell \hookrightarrow 2$, stipulating that $\ell$ will always contain 2. (Invariants like $\ell \hookrightarrow 2$, which are established "dynamically" in the course of a program proof and hold persistently thereafter, are a feature inherited directly from Iris [Jung et al. 2015]. We omit rules for working with them here since they are orthogonal to the focus of this paper.) By LOAD, we easily obtain a lemma

$$
\ell \hookrightarrow 2 \vdash\{\top\} ! \ell\{\text { ret } 2 . \top\}
$$

stating that, under our invariant, dereferencing $\ell$ always returns 2 . To prove that the value $w$ returned by readonly $\ell$ is low-integrity, we apply readonly's specification and have to show that dereferencing $\ell$ returns a low value (which is trivial, by $(\dagger)$ and LiftLit). To prove that the function use $\triangleq \lambda_{\text {. }}$ assert $((! \ell)=2)$ is low-integrity, we apply LiftRec. In this subproof, we have to prove that, under our invariant, the assertion returns a low value (trivial by Assert, $(\dagger)$, LIfTUnit). It remains to show that the pair $(w, u s e)$ is low-integrity given knowledge that both $w$ and use are low-integrity, which is trivial by LifTPAIR. 


\subsection{Metatheory}

OCPL inherits from Iris the basic metatheorem of adequacy for progressive triples (omitted here), which asserts that expressions verified with progressive triples make progress (i.e., do not get stuck) and that, if such expressions terminate, the postconditions of their triples actually hold. OCPL also enjoys an analogous metatheorem for non-progressive triples, which simply drops the progress guarantee. More interestingly, OCPL enjoys the following metatheorems concerning dynamically checked safety properties. The entire metatheory has been verified formally in Coq [OCPL 2017].

Theorem AdequacySafety. If an expression is verified and run in a good state, we can observe that every reachable state is good (i.e., no assertions fail):

$$
\begin{array}{ll}
\{T\} e\{x . T\}_{p} & (e) ;(h, \mathrm{OK}) \longrightarrow^{*} T^{\prime} ;\left(h^{\prime}, g^{\prime}\right) \\
g^{\prime}=\mathrm{OK}
\end{array}
$$

Theorem RobustSafety. Let $A d v C t x$ denote the set of HLA contexts containing neither locations nor assertions. If expression $e$ is closed and has been verified to return only low values, then for every adversarial context $C$, on running $C[e]$ from an initial state, we can observe that every reachable state is good (i.e., no assertions fail):

$$
\frac{C \in A d v C t x \quad e \text { closed } \quad\{T\} e\{x . \text { lowval } x\}_{p} \quad(C[e]) ;(\emptyset, \mathrm{OK}) \longrightarrow^{*} T^{\prime} ;\left(h^{\prime}, g^{\prime}\right)}{g^{\prime}=\mathrm{OK}}
$$

Robust safety captures our informal distinction between "user code" and "untrusted code". Both are written in HLA, but user code must be verified in OCPL and may contain assertion statements, whereas untrusted code need not be verified and is not permitted to contain assertion statements. Untrusted code may also not contain references to memory locations $\ell$, since those are not part of the surface syntax of the language. The theorem says that verified user code can be linked with untrusted code, while remaining safe (i.e., its internal assertion statements will never assert false).

\section{DYNAMIC SEALING}

We now consider one of the oldest and most influential OCPs: dynamic sealing, also called the sealer-unsealer pattern. Originally proposed by Morris [1973], dynamic sealing makes it possible to support data abstraction in the absence of static typing. In this section, we show how OCPL supports compositional reasoning about dynamic sealing. In particular, we show how to implement dynamic sealing in HLA and how to give a compositional specification for this implementation, from which we derive useful specifications for interesting abstractions built on top of it and prove robust safety for representative clients. (We consider an alternative implementation of the OCP, satisfying a slightly weaker specification, in [OCPL 2017, Appendix A]. We also show how ideal specifications for cryptographic signing and encryption primitives can be derived from the specification of dynamic sealing in [OCPL 2017, Appendix B].)

The functionality of dynamic sealing. Morris [1973] introduced dynamic sealing to enforce data abstraction while interoperating with untrusted, potentially ill-typed code. He stipulated a function makeseal for generating pairs of functions (seal, unseal), such that (i) for every value $v$, seal $v$ returns a value $v^{\prime}$ serving as an opaque, low-integrity proxy for $v$; and (ii) for every value $v^{\prime}$, unseal $v^{\prime}$ returns $v$, if $v^{\prime}$ was produced by seal $v$, and otherwise gets stuck. The key point is that this seal-unseal pair supports data abstraction: the client of these functions can freely pass sealed values to untrusted code since they are low-integrity, while at the same time imposing whatever internal invariant it wants on the underlying values that they represent. 


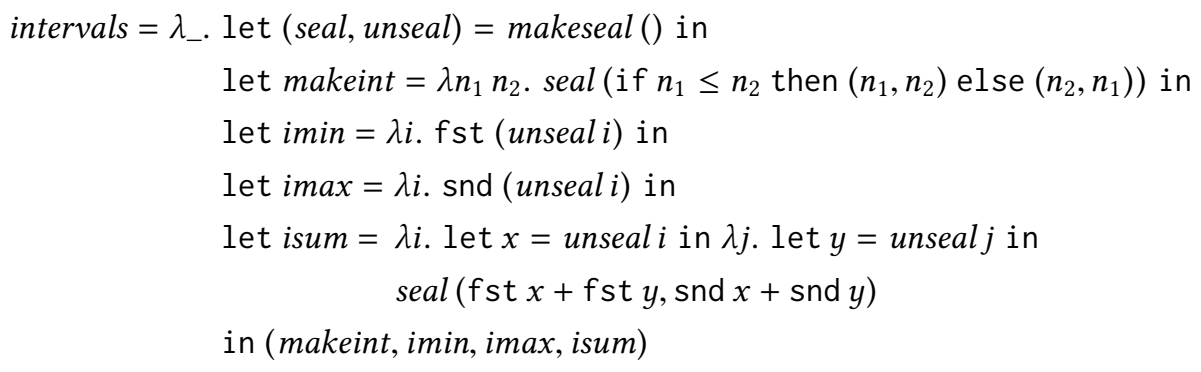

Fig. 4. Library for integer intervals

To see how this is useful, consider a simple client of dynamic sealing, namely Morris's example of a library for integer intervals (Fig. 4). Let $\left[n_{1}, n_{2}\right]$ denote the set $\left\{n_{1}, n_{1}+1, \ldots, n_{2}-1, n_{2}\right\}$. The function intervals returns several interval-manipulating routines: makeint $n_{1} n_{2}$ constructs $\left[\min n_{1} n_{2}, \max n_{1} n_{2}\right], \operatorname{imin}\left[n_{1}, n_{2}\right]$ returns $n_{1}, \operatorname{imax}\left[n_{1}, n_{2}\right]$ returns $n_{2}$, and isum $\left[n_{1}, n_{2}\right]\left[n_{1}^{\prime}, n_{2}^{\prime}\right]$ returns $\left[n_{1}+n_{1}^{\prime}, n_{2}+n_{2}^{\prime}\right]$.

These routines use dynamic sealing to enforce the internal data representation of intervals, namely that the interval $\left[n_{1}, n_{2}\right]$ is represented by the pair $\left(n_{1}, n_{2}\right)$, which is critical to ensuring correctness of the library. In particular, notice that seal and unseal are kept private to the intervals implementation, which means it can enforce that the only values sealed with seal are pairs $\left(n_{1}, n_{2}\right)$ representing intervals (i.e., where $n_{1} \leq n_{2}$ ). Consequently, the imin (resp. imax) function can simply return the first (resp. second) component of its argument after unsealing it, because it knows that, even if the argument comes from untrusted code, so long as the unsealing succeeds, the resulting value will be a pair where the first (resp. second) component represents the lower (resp. upper) bound of the input interval. Furthermore, a client of intervals knows that if it applies both imin and imax to an arbitrary untrusted value $v$, and both operations succeed, producing values $v_{1}$ and $v_{2}$, then $v$ must indeed represent a proper interval $\left[n_{1}, n_{2}\right]$ (with $n_{1} \leq n_{2}$ ), and the resulting values of imin and imax are precisely $v$ 's lower and upper bounds (i.e., with $v_{1}=n_{1}$ and $v_{2}=n_{2}$ ). In this way, dynamic sealing affords programmers the essential functionality of data abstraction, even when interfacing with untrusted code, at the cost of some simple dynamic checks at the boundaries of the abstraction.

Implementation of dynamic sealing. We implement dynamic sealing in HLA as follows. ${ }^{9}$

$$
\begin{aligned}
\text { makeseal } \triangleq \lambda_{-} & \text {let } t b l=\text { ref mapempty in } \\
& \text { let sync }=\text { makesync }() \text { in } \\
& \text { let seal }=\lambda x . \text { let } k=\text { ref }() \text { in } \operatorname{sync}\left(\lambda_{-} . t b l \leftarrow \text { mapinsertnew }(! t b l) k x\right) ; k \text { in } \\
& \text { let unseal }=\lambda k . \text { assume }(\text { isloc } k) ; \operatorname{sync}\left(\lambda_{-} . \text {maplookup }(! t b l) k\right) \text { in } \\
& \text { (seal, unseal })
\end{aligned}
$$

The function makeseal allocates a fresh lookup table $t b l$, which is used to store the mapping between sealed values and their proxies, and access to which is shared by the seal and unseal functions that makeseal returns. To ensure proper synchronization between table accesses during calls to seal and unseal, makeseal also creates a lock via the call to makesync. (The latter returns a higher-order

\footnotetext{
${ }^{9}$ We discuss the straightforward functions makesync, mapempty, mapinsertnew, and maplookup in [OCPL 2017, Appendix F]. Note that maplookup $f k$ gets stuck if location $k$ is not in the domain of the partial function represented by value $f$.
} 
Data abstraction.

MAKeSEALSpec

$\{\top\}$ makeseal ()$\left\{v_{1} v_{2} \gamma\right.$, ret $\left(v_{1}, v_{2}\right)$. isseal $\gamma v_{1} \phi *$ isunseal $\left.\gamma v_{2} \phi\right\}$

SEALSPEC

\{isseal $\gamma s \phi * \phi v\} s v\left\{x^{\prime}\right.$. issealed $\left.\gamma v x^{\prime} \phi\right\}$

UNSEALSPEC

UNSEALANYSPEC

\{isunseal $\gamma u \phi *$ issealed $\left.\gamma v v^{\prime} \phi\right\} u v^{\prime}\{$ ret $v$. T\} $\quad$ isunseal $\gamma u \phi\} u v^{\prime}\left\{x\right.$. issealed $\left.\gamma x v^{\prime} \phi\right\}$ ?

SEALEDINV

issealed $\gamma v v^{\prime} \phi \vdash \phi v$

SEALEDAgree

issealed $\gamma v_{1} v^{\prime} \phi *$ issealed $\gamma v_{2} v^{\prime} \phi \vdash v_{1}=v_{2}$

Low values.

$\begin{array}{lll}\text { SEALEDLow } & \text { SEALLow } & \text { Unseallow } \\ \text { issealed } \gamma v v^{\prime} \phi \vdash \text { lowval } v^{\prime} & \frac{(\forall v) \text { lowval } v \vdash \phi v}{\text { isseal } \gamma s \phi \vdash \text { lowval } s} & \frac{(\forall v) \phi \text { lowval } v}{\text { isunseal } \gamma u \phi \vdash \text { lowval } u}\end{array}$

Fig. 5. Sealing interface (presupposing $\phi$ persistent)

function sync, which ensures mutual exclusion among any expressions $e_{i}$ run under sync $\left(\lambda_{-} . e_{i}\right)$. For more details on makesync, see [OCPL 2017, Appendix F].) An entry [ $k \mapsto v]$ in $t b l$ signifies that location $k$ is a low-integrity proxy for sealed value $v$. To seal a value $v$, we allocate a fresh, low location $k$, extend the table, and return $k$. To unseal a value $v^{\prime}$, we require that $v^{\prime}$ be a location $k$ and then look up $k$ in the table.

To test whether the argument to unseal is a location, we use a primitive unary operator isloc (with boolean return type), as well as an assumption expression. The expression assume $e$ resembles a C- or Java-style assertion: it returns unit if $e$ evaluates to true; otherwise, it gets stuck. It is easy to define assumption expressions using a stuck term abort:

$$
\text { abort } \triangleq 00 \quad \text { assume } e \triangleq \text { if } e \text { then () else abort }
$$

These satisfy the following specifications.

$$
\begin{array}{ll}
\text { АвоRт } & \text { Assume } \\
\{T\} \text { abort }\{x, Q\} & \{P\} e\{x . x=\text { true } * Q\}_{\text {? }} \vdash\{P\} \text { assume } e\{\text { ret }() . Q\}
\end{array}
$$

ABORT follows trivially from APPNREC and gives abort a non-progressive triple because in fact abort always gets stuck and never produces a value; correspondingly, it can also be given an arbitrary postcondition $Q$. Compared to AsSert in $§ 2.2$, Assume (i) uses non-progressive triples since assume $e$ may get stuck, and (ii) only requires the postcondition $Q$ to be shown to hold under the assumption that $e$ returns true.

Specifying dynamic sealing. The motivation for dynamic sealing given above was completely informal. Let us now see how OCPL lets us formalize it.

We specify dynamic sealing in Fig. 5. Rule MAKESEALSPEC for allocating a sealer-unsealer pair ties the two functions it returns to an abstract (logical) name $\gamma$ and representation invariant $\phi$. In particular, the assertions ${ }^{10}$ isseal $\gamma v_{1} \phi$ and isunseal $\gamma v_{2} \phi$ represent knowledge that $v_{1}$ and $v_{2}$ are the seal and unseal functions associated with the sealer-unsealer pair named $\gamma$ with representation invariant $\phi$.

\footnotetext{
${ }^{10}$ In our specifications, predicates whose names start with "is" produce, when fully applied, persistent propositions (and are themselves called persistent predicates). Persistent propositions may be regarded as intuitionistic (technically, they may be freely duplicated). A sealer-unsealer pair's representation invariant $\phi$ must be persistent.
} 
Properties.

\begin{tabular}{|c|c|}
\hline $\begin{array}{l}\text { MinSpec } \\
\{\text { ismin } \gamma\end{array}$ & isinterval $\left.\gamma n_{1} n_{2} i\right\}$ imin $i\left\{\right.$ ret $\left.n_{1} . \top\right\}$ \\
\hline $\begin{array}{l}\text { INTERVALINV } \\
\text { isinterval } \gamma n_{1} n_{2} i \vdash n_{1} \leq n_{2}\end{array}$ & $\begin{array}{l}\text { INTERVALAGREE } \\
\text { isinterval } \gamma n_{1} n_{2} i * \text { isinterval } \gamma n_{1}^{\prime} n_{2}^{\prime} i\end{array}$ \\
\hline
\end{tabular}

Non-progressive triples.

\author{
MinANySPeC \\ $\{$ ismin $\gamma$ imin $\} \operatorname{imin} v\left\{n_{1} n_{2} \text {, ret } n_{1} \text {. isinterval } \gamma n_{1} n_{2} v\right\}_{\text {? }}$ \\ MAXAnySPec \\ $\{$ ismax $\gamma \operatorname{imax}\} \operatorname{imax} v\left\{n_{1} n_{2} \text {, ret } n_{2} \text {. isinterval } \gamma n_{1} n_{2} v\right\}_{\text {? }}$
}

Low values.

$\begin{array}{lll}\text { IntervalLow } & \text { MinLow } & \text { MaxLow } \\ \text { isinterval } \gamma n_{1} n_{2} i \vdash \text { lowval } i & \text { ismin } \gamma \text { imin } \vdash \text { lowval imin } & \text { ismax } \gamma \text { imax } \vdash \text { lowval imax }\end{array}$

Fig. 6. Intervals interface (selected rules-see [OCPL 2017, Appendix C])

Similarly, the assertion issealed $\gamma v v^{\prime} \phi$ represents knowledge that $v^{\prime}$ is a low-integrity proxy for value $v$ (for the sealer-unsealer pair named $\gamma$ with representation invariant $\phi$ ). This relation is functional in $v^{\prime}$-i.e., unsealing the same $v^{\prime}$ twice will produce the same $v$ (SEALEDAgREE)-and implies $\phi v$-i.e., that the unsealed value $v$ must satisfy the representation invariant (SEaledinv). Conversely, to seal a value $v$, one is required to prove $\phi v$ (SealSpec). The progressive triple UnSEALSpec is suitable for use when one already knows that $v$ and $v^{\prime}$ are related; it returns $v$. The non-progressive triple UnSEALANYSPEC returns the value $v$ related to $v^{\prime}$, or gets stuck.

Finally, the three rules at the bottom of Fig. 5 concern the lowval relation. Rule SEALEDLow says that all sealed values are low values-indeed, the whole point of using sealing is to produce low-integrity proxy values-while rules SEALLow and UnSEALLow say when the seal and unseal functions themselves can be considered low values. Intuitively, seal and unseal can be considered low values whenever doing so does not either (i) violate the representation invariant $\phi$ or (ii) result in high-integrity values flowing to a low context. Thus, seal is low so long as any value that is passed to it (i.e., any low value) satisfies $\phi$, and unseal is low so long as any value returned by it (i.e., any value that satisfies $\phi$ ) is low. (We show how to gainfully employ low seal and unseal functions in [OCPL 2017, Appendix B].)

The specification for dynamic sealing from Fig. 5 can be straightforwardly used to derive an analogous specification for the interval-manipulation routines generated by intervals, as we described above. In particular, by choosing the representation invariant $\phi v \triangleq \exists n_{1}, n_{2} . v=\left(n_{1}, n_{2}\right) * n_{1} \leq n_{2}$, we can easily verify the rules shown in Fig. 6 (the full specification for intervals is given in [OCPL 2017, Appendix C]). The assertion isinterval $\gamma n_{1} n_{2} i$ represents knowledge that value $i$ represents interval $\left[n_{1}, n_{2}\right]$ in the instance of the intervals library with abstract name $\gamma$. A value may represent at most one interval (IntervalAgree). A set of progressive triples (e.g., MinSpec) account for our informal specification and are suited to reasoning about values known to represent intervals. A set of non-progressive triples are suited to reasoning about values that might represent intervals. Rule MinAnySpec, for example, says that for any value $v$, imin $v$ might get stuck, but if it terminates with value $\hat{v}$, then $v$ represents some interval with minimum $n_{1}$ and $\hat{v}=n_{1}$. Lastly, there are rules (e.g., IntervalLow and MinLow) expressing the fact that values representing intervals, and the interval 
routines themselves, may be safely shared with untrusted code. Crucial to the soundness of these rules is the fact that the seal function used internally by intervals is not shared with untrusted code (i.e., not low): according to rule SEALLow, in order for seal to be treated as low, the representation invariant $\phi$ would have to be satisfied by all low values, which it clearly is not.

With the spec for intervals in hand, we can readily prove that the following simple but illustrative client of intervals is robustly safe.

$$
\begin{aligned}
\text { client } \triangleq & \text { let cap }=\text { intervals }() \text { in } \\
& \text { let }(\text { makeint, imin, imax, isum })=\text { cap in } \\
& \text { let } \text { check }=\lambda j . \text { assert }(\text { imin } j \leq \operatorname{imax} j) \text { in } \\
& (\text { check, cap })
\end{aligned}
$$

The expression constructs an instance cap of the intervals library, along with a function check which takes an arbitrary value $j$ as its argument and asserts that imin $j$ is no greater than imax $j$. Intuitively, even if client is shared with untrusted code, this assertion must always succeed, because if the applications of imin and imax do not get stuck, it means that $j$ is a proper interval value, whose lower bound is $\leq$ its upper bound.

Formally, this is guaranteed as follows. First, using the spec for intervals, we can prove

$$
\{T\} \text { client }\{x \text {. lowval } x\}
$$

The key step in the proof involves showing that the assertion in check succeeds for arbitrary $j$, which follows directly from MinAnySpec, MaxAnySpec, IntervalAgree, and Intervalinv. Second, we appeal to Theorem RobustSafety from §2.3, which implies that the assertion in check will not fail, even when client is linked with untrusted code.

\section{CARETAKER}

Next, we consider another well-known OCP, the caretaker pattern [Miller and Shapiro 2003; Miller 2006]. This OCP allows verified (trustworthy) code to grant untrusted code access to a highintegrity resource (a high-integrity location or an API that modifies high-integrity locations), and subsequently disable or enable the access at any time. When the caretaker is enabled, untrusted code can access the resource; when the caretaker is disabled, untrusted code cannot access the resource and the verified code has full control over the resource. The caretaker pattern is useful when the verified code wants to ensure that the untrusted code can access the resource only while some invariant holds. Disabling the caretaker allows the verified code to temporarily break the invariant, secure in the knowledge that untrusted code won't be able to access the resource until the caretaker is re-enabled.

In this section, we first implement a caretaker for APIs in HLA, and specify and verify it in OCPL. Then, we use the API caretaker to implement a second caretaker for locations and, again, specify and verify it. Finally, we present a simple client for the location caretaker and establish that it is robustly safe.

API caretaker. The API caretaker allows verified code to share revokable access to any set of functions, which may have side-effects.

$$
\begin{aligned}
\text { makecaretaker } & \triangleq \lambda . \text { let } \text { enabled }=\text { ref false in let sync }=\text { makesync }() \text { in }(\text { sync }, \text { enabled }) \\
\text { wrap } & \triangleq \lambda(\text { sync }, \text { enabled }) f x . \text { sync }\left(\lambda_{-} . \text {assume }(! \text { enabled }) ; f x\right) \\
\text { enable } & \triangleq \lambda(\text { sync }, \text { enabled }) . \text { sync }\left(\lambda_{-} . \text {enabled } \leftarrow \text { true }\right) \\
\text { disable } & \triangleq \lambda(\text { sync }, \text { enabled }) . \text { sync }\left(\lambda_{-} . \text {enabled } \leftarrow \text { false }\right)
\end{aligned}
$$




\author{
ENABLEDEXCLUSIVE \\ enabled $\gamma b_{1} *$ enabled $\gamma b_{2} \vdash \perp$ \\ MAKECARETAKERSPEC \\ $\{\top\}$ makecaretaker ()$\{c t \gamma$, ret $c t$. iscaretaker $\gamma$ ct $R *$ enabled $\gamma$ false $\}$ \\ CANWRAP \\ canwrap $f R \triangleq \forall v$. \{lowval $v * R\} f v\left\{x^{\prime} \text {. lowval } x^{\prime} * R\right\}_{\text {? }}$ \\ WRAPSPEC \\ $\{$ iscaretaker $\gamma$ ct $R *$ canwrap $f R\}$ wrap ct $f\left\{f^{\prime}\right.$. lowval $\left.f^{\prime}\right\}$ \\ ENABLESPEC \\ $\{$ iscaretaker $\gamma$ ct $R *$ enabled $\gamma$ false $* R\}$ enable ct $\{$ ret () . enabled $\gamma$ true $\}$ \\ DisABLeSpec \\ $\{$ iscaretaker $\gamma$ ct $R *$ enabled $\gamma$ true $\}$ disable ct $\{$ ret (). enabled $\gamma$ false $* R\}$
}

Fig. 7. API caretaker interface

The function makecaretaker returns a new caretaker $c t$, which comprises a fresh lock sync, whose purpose is described soon, and a fresh boolean reference enabled. The caretaker is disabled (enabled) when enabled is false (true). The functions enable ct and disable ct enable and disable the caretaker ct by setting enabled appropriately. The function wrap ct $f$ wraps the function $f$ in the caretaker $c t$, returning a function that behaves exactly like $f$ when $c t$ is enabled, and gets stuck when $c t$ is disabled. Additionally, accesses to all functions wrapped in $c t$ are serialized using the lock sync.

To use this interface, verified code creates a caretaker $c t$ and holds it privately. It can then wrap any number of API functions using wrap and disclose the wrapped functions to untrusted code. The untrusted code's access to all those functions can be simultaneously disabled and enabled by calling disable ct and enable ct, respectively.

API caretaker specification. The goal of using the API caretaker is to ensure that untrusted code can access wrapped functions only while some logical resource or invariant $R$ holds on the heap. Our specification of the API caretaker in Fig. 7 formalizes this intuition. The assertion iscaretaker $\gamma$ ct $R$ represents knowledge that value $c t$ is a caretaker with (logical) name $\gamma$ whose wrappers allow (untrusted code) access to wrapped functions only when $R$ holds. The assertion enabled $\gamma b$ represents exclusive ownership of the (verified code's) right to enable and disable all wrappers created using the caretaker named $\gamma$ as well as knowledge that the caretaker is currently enabled (if $b=$ true) or disabled (otherwise).

When applying MakeCAREtakerSpec, one may pick an arbitrary invariant $R$. The spec returns a disabled caretaker tied to $R$ and a fresh name $\gamma$. To enable $c t$, the (verified) code calling enable must establish that $R$ holds (rule EnableSpec). Dually, rule DisableSpec says that disabling $c t$ provides ownership of $R$ to the caller (verified code).

Rule WRAPSPEC is at the heart of our specification. It says that to wrap a function $f$ using $c t$, one has to prove that $f$, when applied to any low value $v$ (possibly provided by the untrusted code), preserves the invariant $R$ and returns a low value. We capture this client responsibility in the precondition (canwrap $f R$ ) on wrap. So, any wrapped function $f$ can assume $R$ in its precondition but it must re-establish $R$ if it terminates (note that the triple for $f v$ in rule CANWrap is nonprogressive). This ensures that while $c t$ is enabled, calls to the wrapped functions by the untrusted 
code preserve $R$. While $c t$ is disabled, verified code can change the state to break $R$ but, prior to re-enabling $c t$, it must re-establish $R$.

The implementation of the API caretaker verifies against this specification. We note that the specification and verification of the API caretaker closely resemble similar efforts for specifying and verifying locks in concurrent programs [Dinsdale-Young et al. 2010]. Based on this observation, we offer a shorter implementation of the API caretaker in [OCPL 2017, Appendix D]. This implementation uses lock release and acquire instead of the boolean reference enabled to enable and disable the caretaker.

The API caretaker is a very general OCP. It can be used to build caretakers for other kinds of resources. As an instance, we show next how to build a caretaker for locations using the API caretaker.

Location caretaker. The location caretaker, defined by the function makelocct below, facilitates revokable and mediated read and write access to a location. Specifically, it ensures that the location is accessed by untrusted code only while some stipulated predicate $\Psi$ holds on the location's contents.

$$
\begin{aligned}
& \text { makelocct } \triangleq \lambda \text { rmon wmon } r . \text { let } c t=\text { makecaretaker }() \text { in } \\
& \text { let read }=\text { wrapct }\left(\lambda_{-} . r m o n(! r)\right) \text { in } \\
& \text { let } \text { write }=\text { wrapct }(\lambda x . r \leftarrow(\text { wmon } x) \text { in } \\
&(c t,(\text { read }, \text { write }))
\end{aligned}
$$

The function makelocct, when applied to a function $f_{\mathrm{r}}$, a function $f_{\mathrm{w}}$, and a location $\ell$, returns a pair $(c t,($ read, write $))$. The API caretaker $c t$ controls whether or not the functions $\{$ read, write $\}$ are enabled. When $c t$ is enabled, the function read reads $\ell$ and filters the read value through $f_{\mathrm{r}}$. Similarly, write writes $\ell$ after filtering the value to be written through $f_{\mathrm{w}}$. Thus, $f_{\mathrm{r}}$ and $f_{\mathrm{w}}$ mediate reads from and writes to $\ell$. Consider, for example, an application write $v$, representing a request to write value $v$ to location $\ell$. If $c t$ is enabled, the application reduces to $\ell \leftarrow\left(f_{\mathrm{w}} v\right)$ and $f_{\mathrm{w}}$ gets to decide what is written.

To use the location caretaker on a location $\ell$, verified code invokes makelocct $f_{\mathrm{r}} f_{\mathrm{w}} \ell$ with appropriate $f_{\mathrm{r}}$ and $f_{\mathrm{w}}$. It holds $\ell$ and the returned API caretaker $c t$ private, but passes read and write to untrusted code. It can then enable and disable access to the protected location using the calls enable $c t$ and disable ct, respectively. While $c t$ is enabled, all reads and writes to $\ell$ are mediated by $f_{\mathrm{r}}$ and $f_{\mathrm{w}}$, respectively.

Location caretaker specification. Our location caretaker specification (Fig. 8) ensures that the value in the protected location $\ell$ satisfies some stipulated predicate $\Psi$ whenever the location is accessible to the untrusted code. The proposition islocct $\gamma c t \ell \Psi$ means that value $c t$ is an API caretaker for location $\ell$ with logical name $\gamma$ and predicate $\Psi$ governing the contents of location $\ell$ when the caretaker is enabled. Logical names and propositions of the form enabled $\gamma b$ serve the same purpose as in the API caretaker interface.

The triple MAKеLосCтSрес specifies the function makelocct and imposes conditions on the function's first two arguments, $f_{\mathrm{r}}$ and $f_{\mathrm{w}}$. Since $f_{\mathrm{r}}$ receives a value read from the protected location, $f_{\mathrm{r}}$ 's input can be assumed to satisfy $\Psi$. Since the output of $f_{\mathrm{r}}$ will be returned to untrusted code, that output must be low. Hence, $f_{\mathrm{r}}$ must transform values satisfying $\Psi$ to low values. Dually, since $f_{\mathrm{w}}$ receives a value from untrusted code and its output is written to the protected location, $f_{\mathrm{w}}$ must transform low values to values satisfying $\Psi$. The postcondition for makelocct says that the returned functions read, write are low, so they can be passed to untrusted code safely. The precondition of enable insists that $\ell$ point to a value $v$ satisfying $\Psi$ before the caretaker is enabled (rule LocCtEnableSpec). 


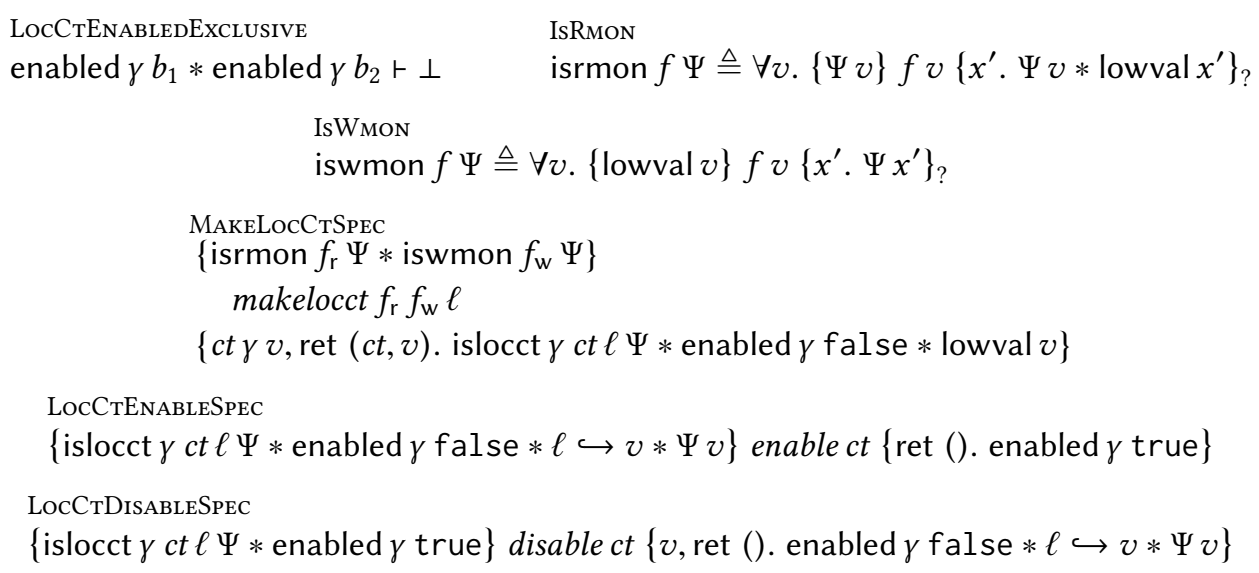

Fig. 8. Location caretaker interface

Dually, the postcondition of disable reveals the fact that $\ell$ points to a value $v$ satisfying $\Psi$ (rule LocCtDisableSpec).

The location caretaker's code satisfies this specification, assuming the specification of the API caretaker (Fig. 7). To illustrate how programs may use a location caretaker, and how such programs may be verified, we consider the following very simple program, client.

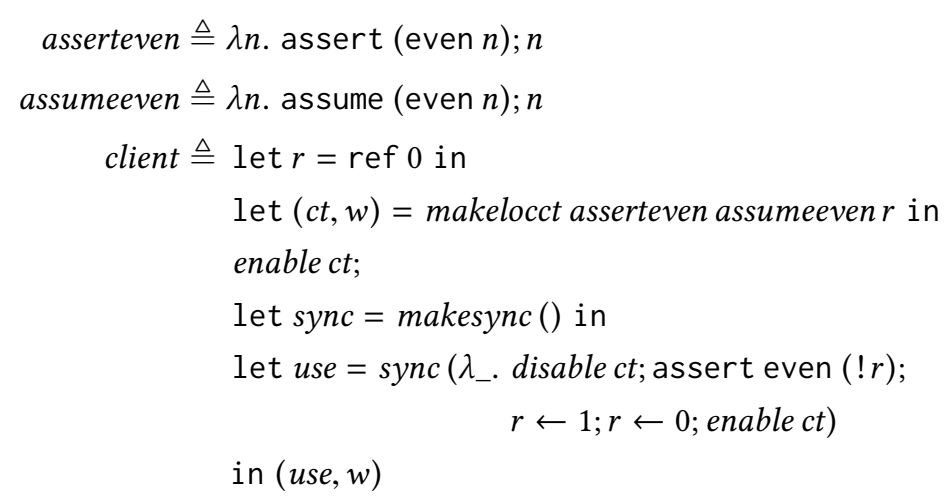

The expression client constructs a reference cell $r$ (initially containing 0 ) and constructs and immediately enables a location caretaker $c t$ serving access to $r$. The goal is to maintain the invariant that untrusted code only sees even numbers in $r$. To ensure this invariant, the location caretaker $c t$ is created with the write filter $f_{\mathrm{w}}=$ assumeeven, which gets stuck unless the value being written is an even number. The read monitor, $f_{\mathrm{r}}=$ asserteven, checks that the value being read from $r$ is indeed even. Additionally, client exposes to its (untrusted) context a function use that (within the scope of an exclusive lock, sync) locally disables the caretaker and temporarily breaks the invariant by writing 1 to $r$.

Using the specification of the location caretaker, we prove that client satisfies the triple

$$
\{\top\} \text { client }\{x \text {. lowval } x\}
$$


By Theorem RobustSafety, client is robustly safe. This means that irrespective of how the (untrusted) context calls use and the wrapped read/write interface $w$, it can never observe a non-even number in $r$ (else, the assertion in asserteven would fail).

\section{MEMBRANE}

When verified (trustworthy) and untrusted code interoperate, a general concern is that values passed from verified to untrusted code may accidentally reveal high-integrity locations. The membrane pattern [Miller 2006; Miller et al. 2008; Google, Inc. 2015] was designed to offset this possibility. A membrane is a bidirectional transformation on values passing from verified to untrusted code and vice versa. In the verified-to-untrusted direction, it sanitizes values to make the extraction of high-integrity locations impossible. A trivial way to implement the membrane is to use the seal function of $\S 3$, which replaces all values with proxies. However, these proxies cannot be used by untrusted code, other than by passing them back to the verified code. To address this shortcoming, the membrane pattern uses a deep inspection of values to selectively hide high-integrity locations inside values, while preserving their overall structure (pairs map to pairs, functions map to functions, etc.). This allows the untrusted code to use the transformed values, while still ensuring that it won't get access to high-integrity locations.

While the specifics of the transformation depend on the language and security objectives of the application, a common core of all membranes is a function that lifts a use case-specific bidirectional transformation on locations to a bidirectional transformation on values. We first define this function, membrane, in HLA below, show its OCPL specification and verify the function. Then, we use this function to implement a specific membrane that we call the public membrane. This membrane replaces each high-integrity location nested in a value with a fresh shadow location and provides the verified code special functions to inspect those shadow locations. This is similar to how the membrane pattern is implemented in Google's Caja, a JavaScript library for securing communication between mutually distrusting domains in web applications [Miller et al. 2008; Google, Inc. 2015]. ${ }^{11}$

Membrane code. The function membrane that forms the common core of the membrane pattern is defined below. This is a higher-order function that takes as its first two arguments two other functions, locout and locin, both of type Loc $\rightarrow$ Val. The function locout defines how locations crossing from verified to untrusted code are transformed by the membrane. Dually, the function locin defines how locations crossing in the other direction, from untrusted to verified code, are transformed. Given these two arguments, membrane locout locin is a function of type $\mathrm{Val} \rightarrow \mathrm{Val}$ that transforms values passing from verified to untrusted code. The function for transforming values passing in the other direction-from untrusted to verified code-is obtained by reversing the arguments to membrane, i.e., it is membrane locin locout.

$$
\begin{aligned}
\text { membrane } \triangleq & \text { rec memb locout locin } x . \\
& \text { let wrap }=\text { memb locout locin in } \\
& \text { if isfun } x \text { then let unwrap }=\text { memb locin locout in } \\
& \quad \lambda y . \text { wrap }(x(\text { unwrap } y)) \\
& \text { else if isloc } x \text { then locout } x \\
& \text { else if islit } x \text { then } x \\
& \text { else if } x=() \text { then }() \\
& \text { else if ispair } x \text { then }(\operatorname{wrap}(\mathrm{fst} x), \operatorname{wrap}(\operatorname{snd} x))
\end{aligned}
$$

\footnotetext{
${ }^{11}$ Google's Caja partly automates the invocation of the membrane transformation by rewriting untrusted code. The automation is orthogonal to the specification and verification of the membrane pattern itself, so we do not model it here.
} 


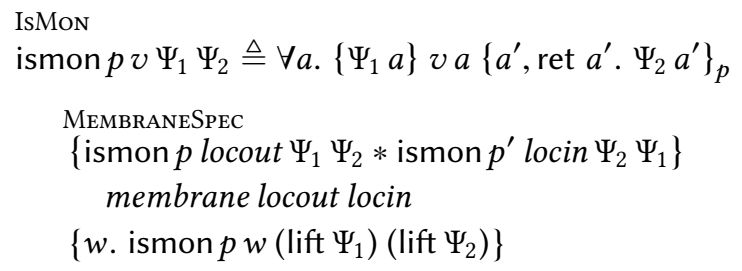

Fig. 9. Membrane interface (presupposing $\Psi_{1}, \Psi_{2}$ persistent)

$$
\begin{aligned}
& \text { else ifinl } x \text { as inl } x^{\prime} \Rightarrow \operatorname{inl}\left(\text { wrap } x^{\prime}\right) \\
& \text { else ifinr } x \text { as inr } x^{\prime} \Rightarrow \operatorname{inr}\left(\text { wrap } x^{\prime}\right) \\
& \text { else assert false } \quad / / \text { i.e., not reached }
\end{aligned}
$$

Internally, membrane recurses on the structure of the value $x$ being transformed. When the value is a location, the result is simply locout $x$. When the value is a literal, it is returned immediately. When the value is a pair, membrane recurses on the two components of the pair. The interesting case arises when the value $x$ is a function. In this case, membrane returns a function, which when applied (by the untrusted code), first recursively applies the membrane to the untrusted argument $y$, then applies the given function $x$ to the transformed argument, and then re-applies the membrane recursively to the result. Importantly, the membrane is applied in the untrusted-to-verified direction to the function argument $y$ and in the verified-to-untrusted direction to the result of the function. Technically, the function $x$ is transformed to wrapox $\circ$ unwrap, where wrap $\triangleq$ memb locout locin and unwrap $\triangleq$ memb locin locout are recursive instantiations of membrane in the verified-to-untrusted and untrusted-to-verified directions, respectively.

Membrane specification. Our specification of membrane formalizes the intuition that membrane lifts transformations on locations to transformations on values. The specification is shown in Fig. 9. The defined predicate ismon $p v \Psi_{1} \Psi_{2}$ means that $v$ is a function that transforms values satisfying the predicate $\Psi_{1}$ to values satisfying the predicate $\Psi_{2}$ (the progress bit $p$ is needed for technical reasons, that readers may ignore). The specification of membrane says that if locout transforms locations satisfying $\Psi_{1}$ to values satisfying $\Psi_{2}$ and locin does the reverse, then membrane locout locin transforms values satisfying lift $\Psi_{1}$ to values satisfying lift $\Psi_{2}$, where lift is the predicate transformer defined in Fig. 3. Hence, membrane really "lifts" the transformation on locations to a transformation on values in a precise technical sense.

This specification of membrane is very general, since it holds for any predicates $\Psi_{1}$ and $\Psi_{2}$. In any use of membrane, these predicates can be instantiated to match what the arguments locout and locin do. (Later, we show a specific instantiation with $\Psi_{2}=$ lowloc.)

Despite the specification's generality, membrane is easily verified against it. In the recursive cases, the proof obligations match the inductive hypotheses precisely because of the concordance between the pattern matches in the definitions of lift (Fig. 3) and membrane.

Public membrane. Next, we describe how the function membrane can be used to construct a specific membrane, which we call the public membrane. This membrane is similar to the membrane used in Google's Caja library [Miller et al. 2008; Google, Inc. 2015]. The public membrane maintains a unique low-integrity shadow location for every high-integrity location that the verified code declares as important. When values cross from verified to untrusted code, all nested high-integrity locations in them are effectively "replaced" with the corresponding low-integrity shadows (this 


$$
\begin{aligned}
\text { makepub } \triangleq \lambda_{-} . & \text {let } t b l=\text { ref bijempty in } \\
& \text { let sync }=\text { makesync }() \text { in } \\
& (\text { sync, } t b l)
\end{aligned}
$$$$
\text { pubout } \triangleq \lambda\left(\text { sync, tbl) } r_{1}\right. \text {. }
$$$$
\operatorname{sync}\left(\lambda_{-} . \text {bijlookup }(! t b l) r_{1}\right)
$$$$
\text { pubin } \triangleq \lambda(\text { sync, tbl }) r_{2} \text {. }
$$$$
\operatorname{sync}\left(\lambda_{-} . \text {bijlookup }(\text { bijinvert }(! t b l)) r_{2}\right)
$$

pubwrap $\triangleq \lambda m$. membrane $($ pubout $m)($ pubin $m)$

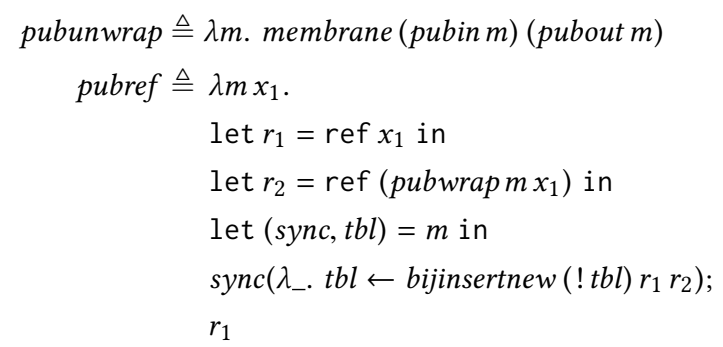

shadowread $\triangleq \lambda m r$. pubunwrapm $(!($ pubout $m r))$

shadowwrite $\triangleq \lambda m r x$. pubout $m r \leftarrow$ pubwrap $m x$

Fig. 10. Public membrane implementation

transformation is implemented using membrane). Thus, the untrusted code only sees shadow locations, not the high-integrity locations. Additionally, the public membrane provides the verified code special functions, shadowread and shadowwrite, to read and write the contents of the shadow locations.

To understand why this is useful, consider a library that allocates an integer reference $\ell$, and shares it with (untrusted) clients as an I/O buffer. Clients are expected to write only positive integers to $\ell$, although the library does not strictly require this and the library's algorithms can execute safely even if the integer is not positive. Over time, many clients of this library have been written. Now, suppose that the library is updated to use different algorithms that really require $\ell$ to always be positive (else they crash). The obvious way to do this would be to rewrite the library to hold $\ell$ private, and to export two closures that read and write $\ell$, the latter only after checking that the value being written is positive. However, this change breaks compatibility with all existing clients, since they must now be rewritten to invoke the new closures to access $\ell$. The public membrane offers a general solution to this problem. Rather than export closures, the library can deploy a public membrane and declare $\ell$ as high-integrity. The membrane consistently replaces $\ell$ with a low-integrity shadow, say $\ell^{\prime}$, for the library's clients. Importantly, the library's clients don't have to change. After a client updates $\ell^{\prime}$ (believing that it updated $\ell$ ), the library can access $\ell^{\prime}$ using shadowread and copy it to $\ell$ if the updated value is a positive integer. Additionally, whenever the library updates $\ell$ internally, it can also copy the update to $\ell^{\prime}$ using shadowwrite. This way, the library can maintain its new invariant and retain complete compatibility with existing clients.

The functions comprising the public membrane are listed in Fig. $10 .^{12}$ The expression makepub() creates a public membrane (consistently denoted $m$ ), which comprises a pair (sync, tbl) where the reference cell $t b l$ contains a finite partial bijection on locations protected by (the lock buried in) sync. When a public membrane's table sends location $\ell_{1}$ to $\ell_{2}$, we say that location $\ell_{1}$ is a (high-integrity) private location and location $\ell_{2}$ its (low-integrity) shadow location. The functions pubout and pubin transform between private and shadow locations by consulting the table. Both functions get stuck on locations not in the table. The functions pubwrap and pubunwrap lift these to transformations between values by applying the function membrane. Effectively, pubwrap replaces all private locations in the value passed to it with their shadow locations and pubunwrap does the opposite.

\footnotetext{
${ }^{12}$ We discuss the straightforward functions makesync, bijempty, bijinsertnew, bijlookup, and bijinvert in [OCPL 2017, Appendix F].
} 


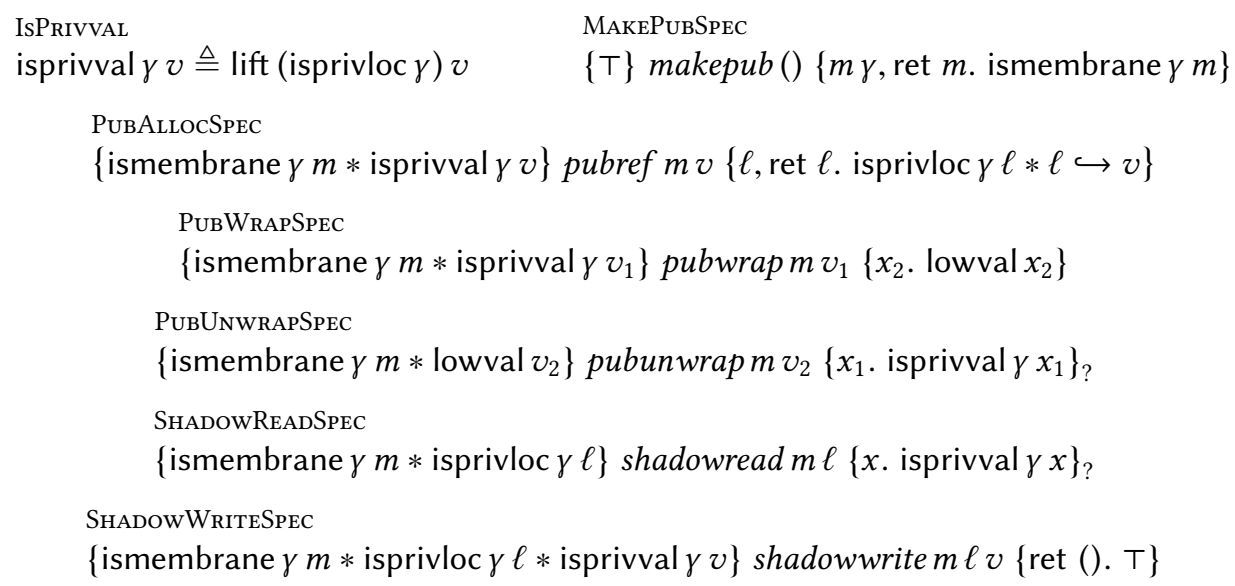

Fig. 11. Public membrane interface

The expression pubref $m v_{1}$ constructs a new private location initially containing value $v_{1}$. In addition, it allocates a shadow location (called $r_{2}$ in the code) that initially contains the low-integrity counterpart of $v_{1}$. It stores an association between the two locations in $m$ 's table. The shadow location $r_{2}$ can be retrieved from the table by applying either pubout or pubwrap to the private location returned by pubref.

The function shadowread inspects a private location's shadow location, converting its lowintegrity contents into a high-integrity value using pubunwrap, whereas shadowwrite updates a private location's shadow location after converting the given value to a low-integrity value using pubwrap.

To use this public membrane interface, the verified code first creates a new public membrane using makepub. Subsequently, it allocates private locations that might flow to untrusted code using the function pubref instead of the language construct ref. Before sending any value to untrusted code, it invokes pubwrap to replace all nested private locations with their shadows. Dually, after receiving any value from untrusted code, it applies pubunwrap to replace nested shadow locations with corresponding private locations. At any point, the verified code can access shadow locations using shadowread and shadowwrite. [OCPL 2017, Appendix E] shows an example of how the public membrane is used.

Public membrane specification. The OCPL specification of the public membrane is shown in Fig. 11. The assertion ismembrane $\gamma m$ associates a public membrane $m$ to a logical name $\gamma$. The abstract predicate isprivloc $\gamma \ell$ represents knowledge that location $\ell$ is a private location for the membrane named $\gamma$, i.e., $\ell$ was previously allocated using pubref on the public membrane named $\gamma$. The assertion isprivval $\gamma v$ is defined as lift (isprivloc $\gamma$ ) $v$ by rule IsPrivval. In particular, the assertion means that any location "extractable" from $v$ is in the domain of $\gamma$ 's table. We call such values $v$ private values. ${ }^{13}$

The specification rules are straightforward, so we explain only some salient points here. The triple for pubref (rule PuBAlLocSpec) says that the application pubref $m v$ returns a location $\ell \hookrightarrow v$ together with the knowledge that $\ell$ is a private location, represented by isprivloc $\gamma \ell$. It subsequently becomes the responsibility of the public membrane's client, who owns $\ell \hookrightarrow v$, to protect any high-integrity

\footnotetext{
${ }^{13}$ Reasoning with private values is fairly straightforward using the definition in Fig. 3 and derived rules like LiftApp (§2.2).
} 
state reachable from $\ell$-for example, by keeping $\ell$ hidden as we do in [OCPL 2017, Appendix E]. The triples PubWrapSpec and PubUnwrapSpec say that the function pubwrap $m$ converts private values to low values, while pubunwrap $m$ does the opposite. In particular, the output of pubwrap, being low, can be freely shared with untrusted code. Also, pubunwrap has a non-progressive triple because unwrapping an arbitrary low value $v_{2}$ may apply pubin $m \ell_{2}$ with some low-integrity location $\ell_{2}$ unknown to $m$, and our implementation of pubin gets stuck in such cases. ${ }^{14}$

The implementation of the public membrane satisfies this specification. As an example, we briefly describe how we verify the pubwrap specification, PuBWrapSPec. First, we prove the following two lemmas showing that for a valid membrane $m$, pubout $m$ and pubin $m$ produce functions that satisfy the predicate ismon.

\section{\{ismembrane $\gamma m\}$ pubout $m\{f$. ismon progress $f$ (isprivloc $\gamma$ ) lowloc $\}$ \\ $\{$ ismembrane $\gamma m$ \} pubin $m\{f$. ismon noprogress $f$ lowloc (isprivloc $\gamma$ ) $\}$}

Next, we instantiate MembraneSpec (Fig. 9) with $\Psi_{1}=$ isprivloc $\gamma, \Psi_{2}=$ lowloc and the two lemmas above to obtain

ismon progress pubwrap (isprivval $\gamma$ ) lowval

The triple PubWrapSpec follows by unfolding the definition of ismon (Fig. 9).

\section{RELATED WORK}

This paper focuses on the formal specification and verification of OCPs. While we know of only rather preliminary prior work in this space, a major point of our paper is to observe that the tools needed for effective verification of OCPs are already to a large extent available-the pieces just need to be assembled properly. In particular, our logic, OCPL, gets significant mileage out of existing verification techniques, notably robust safety and concurrent separation logic. In the following, we discuss related work on robust safety, the (limited amount of) existing work on specification of OCPs, and some other relevant work on object capabilities and ownership types.

Robust safety. The concept of robust safety arose in the context of verifying security protocols that interact with adversaries. Early work used typing to prove "correspondence properties" for cryptographic protocols modeled in the spi calculus [Gordon and Jeffrey 2001]. In their work on the refinement type-checker F7, Bengtson et al. [2011] generalized robust safety to a richer class of integrity properties for a process calculus, RCF, with higher-order state. We inherit from their work the basic idea of using a notion of low-integrity values and proving robust safety, but the approaches differ greatly in detail. First, we show how to apply this idea to OCPs, a completely different domain. Second, we show how low-integrity values are directly encodable in modern separation logics, using a simple logical relation.

Object capabilities. There has been only very preliminary work on specifying and verifying functional properties of object capabilities and OCPs. In his seminal paper on dynamic sealing, Morris [1973] proposed informal reasoning principles for programmers using dynamic sealing, but did not prove anything formally. Drossopoulou et al. [2015a] proposed predicates modeling trust and risk and used those predicates to specify a capability-based escrow exchange example [Miller et al. 2013]. They focused on this one example, whereas we develop general specifications for several OCPs. Further, they focus on syntactic specifications and do not define the semantics of their predicates. A subsequent manuscript [Drossopoulou et al. 2015b] bridges this gap to the

\footnotetext{
${ }^{14}$ For simplicity, we do not specify the functionality of pubwrap and pubunwrap beyond "returns a low, resp., private value". More useful specifications would allow one to prove that, say, after wrapping private location $\ell_{1}$ twice and obtaining values $v_{2}$ and $v_{2}^{\prime}$, there exists a low location $\ell_{2}$ such that $v_{2}=\ell_{2}=v_{2}^{\prime}$.
} 
semantics, but their Hoare logic seems inadequate for the examples we consider, e.g., it lacks a rule for dynamic allocation.

Perhaps the most closely related work to our own is that of Devriese et al. [2016]. As discussed in the introduction, they use a Kripke logical relation and a meta-property called effect parametricity to verify integrity properties for several examples of capability-wrapped user code in a language with higher-order state. There are several points of difference between our work and theirs. First, we work in a concurrent separation logic rather than directly in a low-level logical relation. As a result, in addition to being able to conduct our proofs at a much higher level of abstraction, we can give compositional specifications for higher-order object capability patterns (i.e., libraries), whereas they only verify specific programs that use object capabilities. We also exploit the notion of robust safety in verifying integrity properties of code that uses OCPs, whereas corresponding arguments only appear implicitly in their proofs. On the other hand, they develop semantic variants of the so-called "reference graph properties" from the literature on object capabilities. These properties are important but they are also orthogonal to verification and, hence, we do not examine them Last but not least, our proofs are machine-checked in Coq.

While specifying OCPs has received little attention so far, there has been a lot of other work on object capabilities and OCPs. Responding to renewed interest in dynamic sealing, Sumii and Pierce [2004] propose a bisimulation for proving contextual equivalences in a language with a dynamic sealing primitive. Roughly, the examples they consider are pairs of expressions-"modules" implementing the same "interface" using sealing-and the question they study is whether those expressions are indistinguishable, even if their internal representations differ. Bengtson et al. [2011] use dynamic sealing to define ideal implementations of cryptographic operations in RCF. They offer no general specifications for dynamic sealing analogous to the specification in $\S 3$, but derive instances of such a specification as needed. Van Cutsem and Miller [2013, §§4.3-4.4] describe a variant of our public membrane, which they use to implement proxy objects supporting socalled language invariants (such as the reasoning enabled, in JavaScript, by the ability to "freeze" object properties). Their focus is on the use of such membranes as an implementation technique, rather than on verification. Spiessens and Van Roy [2005]; Spiessens [2007] and Murray [2010] use, respectively, model- and refinement-checking tools to establish certain safety and liveness properties of abstract models of object capability systems, including some OCPs. To take one small example, Murray shows that a specific model of an unsealing operation does not reveal a sealed value unless a capability to that sealed value was passed (possibly indirectly) to the unsealing operation by the context. Although useful, such properties cannot be directly used to verify clients of the OCPs. In contrast, our goal is very different: we write compositional specifications for concrete implementations of OCPs and our specifications can be directly used to verify clients.

Ownership types. There are interesting similarities between the core mechanisms of OCPL and prior work on ownership types [Clarke et al. 2013]. In their seminal paper, Clarke et al. [1998] proposed a type system based on what came to be called owners-as-dominators. Roughly, the idea is to impose an ownership relation on objects, separating a public owner object from its hidden representation objects and ensuring that an owner mediates all outside access to its representation. Clarke et al. then proved that well-typed code preserves an invariant classifying data and enforcing isolation according to owners-as-dominators. In a similar vein, OCPL classifies locations as highintegrity (private) vs. low-integrity (public), and enforces that high-integrity locations cannot be accessed from low-integrity locations. However, OCPL is not a static type system, but rather a logic for verifying the enforcement of integrity properties in a dynamically-typed setting. Moreover, although OCPL requires that low locations contain low values, it does not stipulate any particular ownership discipline (like owners-as-dominators) for how the user should achieve this. 
Other researchers have proved deeper semantic properties of owners-as-dominators. Banerjee and Naumann [2005a,b] prove relational parametricity theorems, using logical relations as a proof technique for showing that one representation may be safely replaced by another. Patrignani et al. [2011] show that an extension of owners-as-dominators can be used to prove relational secrecy properties for the join calculus, based on a distinction between high- and low-integrity values. In OCPL, we define low-integrity values using a simple (implicitly step-indexed) logical relation, and (like Patrignani et al.) our key meta-theorem concerns the interaction of user code with untyped, untrusted code. However, despite these similarities to prior work, our concrete goals are very different: rather than reasoning about relational properties, we focus on verifying functional specifications of OCPs and the robust safety of capability-wrapped code.

\section{CONCLUSION AND FUTURE WORK}

Object capability patterns (OCPs) enable programmers to enforce invariants on the private state of their objects, even when those objects are passed to untrusted code. In principle, this should make it easier to write secure and correct programs, but in practice, programmers may use OCPs incorrectly, resulting in subtle security flaws. In this paper, we develop OCPL, the first logic for compositionally specifying and verifying OCPs. We deploy it in reasoning about both implementations and representative clients of several well-known OCPs, in the context of a simple but expressive programming language with higher-order state.

We believe that using robust safety and separation logic to reason about OCPs scales to much richer settings. The Firefox web browser, for example, uses an automatic and significantly more sophisticated membrane pattern to enforce the so-called same-origin policy [Mozilla 2016; Barth 2011]. In ongoing work, we are exploring an extension of OCPL to reason about this system. Furthermore, while the Iris proof mode enables relatively high-level Coq proofs, such manual proofs are nonetheless often tedious and routine. Additional research is needed to scale such proofs up to realistic languages and improve automation.

\section{ACKNOWLEDGMENTS}

This research was supported in part by a Microsoft Research PhD Scholarship, and in part by a European Research Council (ERC) Consolidator Grant for the project "RustBelt", funded under the European Union's Horizon 2020 Framework Programme (grant agreement no. 683289). We wish to thank Andrea Fischer and the OOPSLA reviewers for extremely helpful and detailed feedback.

\section{REFERENCES}

Martín Abadi. 1999. Secrecy by typing in security protocols. F. ACM 46, 5 (Sept. 1999), 749-786.

Andrew W. Appel, Paul-André Melliès, Christopher D. Richards, and Jérôme Vouillon. 2007. A very modal model of a modern, major, general type system. In Proceedings of the 34th Annual ACM SIGPLAN-SIGACT Symposium on Principles of Programming Languages (POPL '07). 109-122.

Anindya Banerjee and David A. Naumann. 2005a. Ownership confinement ensures representation independence for object-oriented programs. 7. ACM 52, 6 (Nov. 2005), 894-960.

Anindya Banerjee and David A. Naumann. 2005b. State based ownership, reentrance, and encapsulation. In Proceedings of the 19th European Conference on Object-Oriented Programming (ECOOP '05). 387-411.

Adam Barth. 2011. The Web origin concept. RFC 6454. https://www.rfc-editor.org/rfc/rfc6454.txt

Jesper Bengtson, Karthikeyan Bhargavan, Cédric Fournet, Andrew D. Gordon, and Sergio Maffeis. 2011. Refinement types for secure implementations. ACM Trans. Program. Lang. Syst. 33, 2 (Feb. 2011), 8:1-8:45.

Lars Birkedal, Bernhard Reus, Jan Schwinghammer, Kristian Støvring, Jacob Thamsborg, and Hongseok Yang. 2011. Stepindexed Kripke models over recursive worlds. In Proceedings of the 38th Annual ACM SIGPLAN-SIGACT Symposium on Principles of Programming Languages (POPL '11). 119-132.

Dave Clarke, Johan Óstlund, Ilya Sergey, and Tobias Wrigstad. 2013. Ownership types: A survey. In Aliasing in ObjectOriented Programming: Types, Analysis, and Verification, Dave Clarke, James Noble, and Tobias Wrigstad (Eds.). Springer 
LNCS 7850, 15-58.

David G. Clarke, John M. Potter, and James Noble. 1998. Ownership types for flexible alias protection. In Proceedings of the 13th ACM SIGPLAN Conference on Object-oriented Programming, Systems, Languages, and Applications (OOPSLA '98). $48-64$.

Douglas Crockford. 2008. Making JavaScript safe for advertising. (2008). Retrieved April 2017 from http://www.adsafe.org/

Dominique Devriese, Lars Birkedal, and Frank Piessens. 2016. Reasoning about object capabilities with logical relations and effect parametricity. In IEEE European Symposium on Security and Privacy (EuroS\&P). 147-162.

Thomas Dinsdale-Young, Mike Dodds, Philippa Gardner, Matthew J. Parkinson, and Viktor Vafeiadis. 2010. Concurrent abstract predicates. In Proceedings of the 24th European Conference on Object-Oriented Programming (ECOOP '10). 504-528.

Sophia Drossopoulou, James Noble, and Mark S. Miller. 2015a. Swapsies on the internet: First steps towards reasoning about risk and trust in an open world. In Proceedings of the 10th ACM Workshop on Programming Languages and Analysis for Security (PLAS '15). 2-15.

Sophia Drossopoulou, James Noble, Mark S. Miller, and Toby Murray. 2015b. Reasoning about risk and trust in an open world. Technical Report ECSTR-15-08. Victoria University of Wellington.

Matthias Felleisen and Robert Hieb. 1992. The revised report on the syntactic theories of sequential control and state. Theor. Comput. Sci. 103, 2 (Sept. 1992), 235-271.

Google, Inc. 2015. Caja membrane implementation. (Feb. 2015). https://github.com/google/caja/blob/master/src/com/google/ caja/plugin/taming-membrane.js

Andrew D. Gordon and Alan Jeffrey. 2001. Authenticity by typing for security protocols. In Proceedings of the 14th IEEE Workshop on Computer Security Foundations (CSFW'01). 145-159.

Ralf Jung, Robbert Krebbers, Lars Birkedal, and Derek Dreyer. 2016. Higher-order ghost state. In Proceedings of the 21st ACM SIGPLAN International Conference on Functional Programming (ICFP '16). 256-269.

Ralf Jung, Robbert Krebbers, Jacques-Henri Jourdan, Aleš Bizjak, Lars Birkedal, and Derek Dreyer. 2017. Iris from the ground up: A modular foundation for higher-order concurrent separation logic. (2017). Submitted for publication.

Ralf Jung, David Swasey, Filip Sieczkowski, Kasper Svendsen, Aaron Turon, Lars Birkedal, and Derek Dreyer. 2015. Iris: Monoids and invariants as an orthogonal basis for concurrent reasoning. In Proceedings of the 42nd Annual ACM SIGPLAN-SIGACT Symposium on Principles of Programming Languages (POPL '15). 637-650.

Robbert Krebbers, Ralf Jung, Aleš Bizjak, Jacques-Henri Jourdan, Derek Dreyer, and Lars Birkedal. 2017a. The essence of higher-order concurrent separation logic. In Proceedings of the 26th European Symposium on Programming (ESOP '17). 696-723.

Robbert Krebbers, Amin Timany, and Lars Birkedal. 2017b. Interactive proofs in higher-order concurrent separation logic. In Proceedings of the 44th ACM SIGPLAN Symposium on Principles of Programming Languages (POPL '17). 205-217.

Adrian Mettler, David Wagner, and Tyler Close. 2010. Joe-E: A security-oriented subset of Java. In Proceedings of the Network and Distributed System Security Symposium (NDSS '10).

Mark Samuel Miller. 2006. Robust Composition: Towards a Unified Approach to Access Control and Concurrency Control. Ph.D. Dissertation. Johns Hopkins University.

Mark S. Miller, Tom Van Cutsem, and Bill Tulloh. 2013. Distributed electronic rights in JavaScript. In Proceedings of the 22nd European Conference on Programming Languages and Systems (ESOP '13). 1-20.

Mark S. Miller, Chip Morningstar, and Bill Frantz. 2000. Capability-based financial instruments. In Proceedings of the 4th International Conference on Financial Cryptography (FC '00). 349-378.

Mark S. Miller, Mike Samuel, Ben Laurie, Ihab Awad, and Mike Stay. 2008. Caja: Safe active content in sanitized JavaScript. (June 2008). http://citeseerx.ist.psu.edu/viewdoc/summary?doi=10.1.1.306.6704 Unpublished draft.

Mark S. Miller and Jonathan S. Shapiro. 2003. Paradigm regained: Abstraction mechanisms for access control. In Advances in Computing Science - ASIAN 2003 Programming Languages and Distributed Computation, 8th Asian Computing Science Conference (ASIAN '03). Springer LNCS 2896, 224-242.

James H. Morris, Jr. 1973. Protection in programming languages. Commun. ACM 16, 1 (Jan. 1973), 15-21.

Mozilla. 2016. Script security. (Aug. 2016). https://developer.mozilla.org/en-US/docs/Mozilla/Gecko/Script_security Overview of the Firefox membrane.

Toby Murray. 2010. Analysing the Security Properties of Object-Capability Patterns. Ph.D. Dissertation. Hertford College.

OCPL 2017. Long version of this paper (with appendices) and Coq development. (Sept. 2017). Available at the Iris project website at http://iris-project.org.

Marco Patrignani, Dave Clarke, and Davide Sangiorgi. 2011. Ownership types for the join calculus. In Proceedings of the foint 13th IFIP WG 6.1 and 30th IFIP WG 6.1 International Conference on Formal Techniques for Distributed Systems (FMOODS'11/FORTE'11). 289-303.

Joe Gibbs Politz, Arjun Guha, and Shriram Krishnamurthi. 2014. Typed-based verification of Web sandboxes. f. Comput. Secur. 22, 4 (July 2014), 511-565.

Alfred Spiessens. 2007. Patterns of Safe Collaboration. Ph.D. Dissertation. Universite catholique de Louvain. 
Fred Spiessens and Peter Van Roy. 2004. The Oz-E project: Design guidelines for a secure multiparadigm programming language. In Proceedings of the Second International Conference on Multiparadigm Programming in Mozart/Oz (MOZ '04). 21-40.

Fred Spiessens and Peter Van Roy. 2005. A practical formal model for safety analysis in capability-based systems. In Proceedings of the 1st International Conference on Trustworthy Global Computing (TGC '05). 248-278.

Marc Stiegler and Mark Miller. 2006. How Emily tamed the Caml. Technical Report HPL-2006-116. HP Laboratories.

Eijiro Sumii and Benjamin C. Pierce. 2004. A bisimulation for dynamic sealing. In Proceedings of the 31st ACM SIGPLANSIGACT Symposium on Principles of Programming Languages (POPL '04). 161-172.

Tom Van Cutsem and Mark S. Miller. 2013. Trustworthy proxies: Virtualizing objects with invariants. In Proceedings of the 27th European Conference on Object-Oriented Programming (ECOOP '13). 154-178. 